\title{
Nanoscale
}

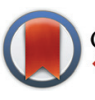

CrossMark

Cite this: Nanoscale, 2016, 8, 5959

\section{Silver nanoparticles confined in carbon nanotubes: on the understanding of the confinement effect and promotional catalysis for the selective hydrogenation of dimethyl oxalate $\uparrow$}

\author{
Jianwei Zheng, Xinping Duan, * Haiqiang Lin, Zhengqiang Gu, Huihuang Fang, \\ Jianhui Li and Youzhu Yuan*
}

\begin{abstract}
A confined Ag nanomaterial in the channels of herringbone multi-walled carbon nanotubes (Ag-in/hCNT) was effectively prepared. The space restriction induces morphological changes of $\mathrm{Ag}$ nanoparticles into rough nanowires with an estimated aspect ratio of $60: 8(\mathrm{~nm} / \mathrm{nm})$. Dihydrogen activation is enhanced through the vacancy-enriched wire-like Ag nanocatalyst, as well as the confinement effect. The grain boundaries of $\mathrm{Ag}$ and rolled-up graphene layers of CNTs are speculated to play vital roles in the diffusion of activated hydrogen species. The Ag-in/hCNT catalyst exhibits an activity that is three times higher than that of Ag nanoparticles located on the CNT exterior walls in DMO hydrogenation. This finding may insinuate that interplanar spaces provide available access to the external surface of CNTs. Designed experiments further confirm the importance of herringbone CNTs with higher reaction rate than parallel CNTs, and confined Ag produces considerably more activated hydrogen species, thereby benefiting the reduction of surface copper nanoparticles or DMO molecules during hydrogenation. This paper presents a study of the effective utilization of hydrogen over herringbone CNT confined $\mathrm{Ag}$ and an understanding of the confinement and promotional catalytic effects.
\end{abstract}

Received 5th December 2015 Accepted 12th February 2016

DOI: $10.1039 / \mathrm{c} 5 \mathrm{nr} 08651 \mathrm{e}$

www.rsc.org/nanoscale particularly useful in integrating the properties of the two components, as abundant stereoelectronic orbits allow a strong electronic effect, affecting the absorption behavior of reactants and supported metals. ${ }^{6}$

In addition, a special long-range and one-dimensional channel bestows CNTs with particular properties and widens their applications. ${ }^{6,7}$ One of the most prominent examples is the utilization of the interior surface of CNTs to encapsulate metals for heterogeneous catalysis. The encapsulated metals were found to exhibit excellent catalytic performance in ethanol synthesis, CO oxidation, and Fischer-Tropsch synthesis. ${ }^{8-10}$ In terms of the confinement effect, the interaction of reactants and products with CNT walls, optimized redox properties of metal catalysts within the CNTs, and chemical reactions over metallic active sites confined inside the CNT channels can be expected to yield different physiochemical behaviors. Reaction issues involving activity, selectivity, and stability can be improved through reconstruction of the encapsulated metallic CNT composites. ${ }^{6}$ For instance, Pd NPs were effectively introduced inside multi-walled CNTs with 5-10 nm inner diameter channels for benzene hydrogenation. ${ }^{11}$ The catalytic activity of confined $\mathrm{Ag}$ was considerably higher than that over catalysts supported by activated carbon (AC) and $\mathrm{Y}$ zeolite, even though multi- 
walled CNTs possess markedly smaller specific surface areas than AC and Y zeolite. Pd NPs deposited inside multi-walled CNTs were also utilized as catalysts for the selective hydrogenation of cinnamaldehyde, and the hydrogenation selectivities towards the $\mathrm{C}=\mathrm{C}$ and $\mathrm{C}=\mathrm{O}$ bonds were significantly different from those over the conventional catalyst with similar conversion. ${ }^{12}$ Depending on the orientation angles of rotation of the graphitic layers relative to the tube axis, multi-walled CNTs can be distinguished as parallel-type and herringbone-type. Despite the widespread use of multi-walled CNTs, the importance of the rolled-up layer structure and the effect and mechanism of confinement on the catalytic performance are barely discussed.

More recently, a significant integrated technology that involves the coupling of syngas-derived methanol with $\mathrm{CO}$ to form dimethyl oxalate and subsequent hydrogenation to yield methyl glycolate (MG) and ethylene glycol (EG) has garnered considerable attention worldwide. ${ }^{13-15}$ Although the second step of EG production has been successfully scaled up into industrial applications, MG production has been neglected. MG is an important fine chemical with more added value in comparison with EG. It is widely used in the synthesis of numerous pharmaceutical products, fine chemicals, and perfumes. These MG-derived products are biodegradable and environmentally friendly. Therefore, MG presents an enormous potential market. However, the main difficulty of MG synthesis through the DMO intermediate approach lies in the design of effective catalysts with high reactivity and selectivity. Supported copper catalysts are preferentially used for DMO hydrogenation to EG and ethanol. ${ }^{13-16}$ However, the mono-hydrogenated (unilateral hydrogenated) product, MG, is usually inferior over copper catalysts because of the high thermodynamic constant of the subsequent hydrogenation of MG to EG. ${ }^{17}$ Silica oxide-supported Ag catalysts were recently reported to possess high MG yield for the chemoselective hydrogenation of DMO to MG. ${ }^{1,17,18}$ The interaction of $\mathrm{Ag}$ with multi-walled CNTs and the implication of confinement in hydrogenation have been scarcely investigated.

In this work, confined/unconfined $\mathrm{Ag}$ catalysts in different kinds of CNTs were fabricated and applied in DMO hydrogenation to $\mathrm{MG}$ for the comparison of dihydrogen activation capacity. Unlike the previously reported confined metal CNT hybrid catalysts, ${ }^{6-12}$ the filling Ag exists in the morphology of nanowires inside the CNT channel. Unexpectedly, these confined $\mathrm{Ag}$-in/hCNT catalysts were found to exhibit superior activity and selectivity, even though blocking of CNTs may hinder the accessibility of the metals in the inner part of the channels to reactants and significant diffusion resistances for products to exhaust out of the channels. For comparison, $\mathrm{Ag}$ catalysts supported on various carriers were prepared to investigate the interaction of $\mathrm{Ag}$ NPs and supports and the hydrogenation mechanism. Comprehensive characterization was performed through transmission electron microscopy (TEM), hydrogen temperature-programmed reduction $\left(\mathrm{H}_{2}\right.$-TPR), X-ray diffraction (XRD), and chemisorption analysis to correlate the geometrical and surface electronic structure with catalytic performance. Finally, a plausible mechanism of hydrogen transfer was proposed.

\section{Experimental}

\subsection{Synthesis of materials}

2.1.1 Preparation of confined Ag-in/hCNT. Classic volumetric impregnation was adopted to fabricate Ag-in/hCNT catalysts. Typically, $0.7 \mathrm{~mL}$ of silver nitrate $\left(\mathrm{AgNO}_{3}\right)$ and $1.0 \mathrm{~g}$ of CNTs adjusted to yield $10 \mathrm{wt} \%$ Ag-loaded catalysts were dissolved in deionized water with one or two drops of $68 \mathrm{wt} \%$ nitric acid solution. The acidic CNTs and silver solution were quickly mixed and subjected to aging at room temperature for $10 \mathrm{~h}$. Prior to calcination in air at $523 \mathrm{~K}$ for $4 \mathrm{~h}$, the obtained powder was dried at $383 \mathrm{~K}$ in the dark. In this case, an equal volume of solution and porous volume of CNT were used. Most of the aqueous solution was drawn into the CNT channels by capillary forces. The following heat treatment facilitated the deposition of $\mathrm{Ag}$ in large part onto the interior surface of the CNT channels. To reduce the outside Ag content, we performed further ultrasonic-aid washing, filtration and drying. It has been reported that the Ag NPs at the outside of CNTs could be detached if the samples were simply immersed in distilled water, since the adhesion of the Ag NPS to CNTs is not strong. ${ }^{19}$ As a result, most of Ag NPs were located in the CNT channels. Prior to catalytic evaluation, the solid catalyst precursors were reduced at $573 \mathrm{~K}$ at temperature ramping rates of $2 \mathrm{~K} \mathrm{~min}^{-1}$ by $5 \% \mathrm{H}_{2}-95 \% \mathrm{~N}_{2}$ gas for $4 \mathrm{~h}$, yielding the samples denoted as Ag-in/hCNT. The inductively coupled plasma-optical emission spectroscopy (ICP-OES) indicated that the actual $\mathrm{Ag}$ content was $8.1 \mathrm{wt} \%$. A similar method was used to prepare $\mathrm{Ag}$ catalysts using different carriers, including $\mathrm{SiO}_{2}, \mathrm{H}_{2} \mathrm{SiO}_{3}, \mathrm{Al}_{2} \mathrm{O}_{3}, \mathrm{SBA}-15$, and other carbon materials, with a theoretical Ag content of $10 \mathrm{wt} \%$.

2.1.2. Preparation of supported Ag-out/hCNT. Ag-out/hCNT was prepared by the excessive impregnation method. Excess amounts $(500 \mathrm{~mL})$ of $\mathrm{AgNO}_{3}$ solution were used to disperse $1.0 \mathrm{~g}$ of CNTs. CNT and $\mathrm{AgNO}_{3}$ solutions were stirred overnight at $333 \mathrm{~K}$ and subsequently dried at $383 \mathrm{~K}$. Only a small amount of $\mathrm{Ag}$ species may be deposited onto the interior surface of CNTs as most of the $\mathrm{AgNO}_{3}$ solution remained outside of CNT channels. The dried precipitate was then calcined for $4 \mathrm{~h}$ at $523 \mathrm{~K}$ and reduced at $573 \mathrm{~K}$ at temperature ramping rates of $2 \mathrm{~K} \mathrm{~min}^{-1}$ by $5 \% \mathrm{H}_{2}-95 \% \mathrm{~N}_{2}$ gas for $4 \mathrm{~h}$, which afforded the samples denoted as $\mathrm{Ag}$-out $/ h \mathrm{CNT}$. ICP-OES indicated that the $\mathrm{Ag}$ loading was $8.9 \mathrm{wt} \%$, which is slightly larger than that of Ag-in/hCNT. A similar process was used to prepare the Cu-out $/ h \mathrm{CNT}$ precursor.

2.1.3. Preparation of Cu-out-Ag-in/hCNT. Prior to impregnation of copper species onto the CNTs, Ag-in/hCNT was calcined at $873 \mathrm{~K}$ under argon flow. The obtained powder was then soaked in the $\mathrm{Cu}\left(\mathrm{NO}_{3}\right)_{2}$ solution. The $\mathrm{CNT}$ and $\mathrm{Cu}\left(\mathrm{NO}_{3}\right)_{2}$ solutions were stirred overnight at $333 \mathrm{~K}$ and dried at $383 \mathrm{~K}$. The dried precipitate was then calcined for $2 \mathrm{~h}$ at $523 \mathrm{~K}$ and reduced at $573 \mathrm{~K}$ at temperature ramping rates of $2 \mathrm{~K} \mathrm{~min}^{-1}$ by $5 \% \mathrm{H}_{2}-95 \% \mathrm{~N}_{2}$ gas for $4 \mathrm{~h}$, yielding the samples denoted as Cu-out-Ag-in $/ h \mathrm{CNT}$.

\subsection{Physicochemical characterization}

2.2.1 BET. The physical properties of the porous structures were determined using $\mathrm{N}_{2}$ adsorption-desorption measure- 
ments under liquid nitrogen temperature with a Micromeritics Tristar 3020 system. The sample was initially outgassed at $573 \mathrm{~K}$ for $3 \mathrm{~h}$ under vacuum before nitrogen adsorption. Subsequently, the vessel was soaked in liquid nitrogen. The surface area was evaluated by applying the Brunauer-EmmettTeller (BET) method.

2.2.2 TEM. TEM images were obtained by a Philips Analytical FEI Tecnai 30 electron microscope equipped with an ultra high-resolution pole piece and operated at an acceleration voltage of $300 \mathrm{kV}$. Catalyst powders were reduced under a flow of $5 \% \mathrm{H}_{2}-95 \% \mathrm{~N}_{2}$ gas in a sealed flask. The reduced gas was replaced as $\mathrm{N}_{2}$, and the sample was cooled to room temperature, followed by injecting ethanol into the flask, which was sealed thereafter. The sample was ultrasonically dispersed in the sealed flask without air exposure. The as-obtained suspension was then dropped and dried on a holey $\mathrm{Cu}$ grid supported by carbon films.

2.2.3 XRD. A PANalytical X'pert Pro Super X-ray diffractometer was operated for powder XRD analysis using $\mathrm{Cu} \mathrm{K}_{\alpha}$ radiation $(\lambda=0.15418 \mathrm{~nm})$. As the confined $\mathrm{Ag}$ nanowires are unsuitable for calculations of the crystallite size in a spherical model, crystallite sizes were calculated using the Scherrer equation.

2.2.4 $\mathbf{H}_{2}$ chemisorption. Chemisorption uptakes of adsorbates $\left(\mathrm{H}_{2}\right)$ on catalysts were measured on a Micromeritics ASAP $2020(\mathrm{M}+\mathrm{C})$ analyzer using the static volumetric method. A measured amount of catalyst (minimum of $500 \mathrm{mg}$ ) sample was reduced under a flow of high-purity dihydrogen at $573 \mathrm{~K}$ for $2 \mathrm{~h}$ and then evacuated for $1 \mathrm{~h}$ to ensure the complete removal of chemisorbed species. Under a precisely controlled temperature of $308 \mathrm{~K}$, an initial isotherm was measured in a pressure range of 100-600 Torr. A $30 \mathrm{~min}$ evacuation at $308 \mathrm{~K}$ was conducted to remove the reversibly adsorbed adsorbate molecules on the catalyst surface prior to measuring a repetitive isotherm under the same conditions as the initial one. The difference between the initial and repetitive isotherms reflects the uptake of the irreversibly chemisorbed adsorbates on catalysts.

2.2.5 $\mathbf{H}_{2}$-TPR. $\mathrm{H}_{2}$-TPR profiles for the as-calcined samples were measured using a Micromeritics Autochem II 2920 instrument. Prior to reduction, the catalyst was first treated under $\mathrm{Ar}$ flow at $523 \mathrm{~K}$ for $1 \mathrm{~h}$. After cooling to room temperature, a flow of $5 \% \mathrm{H}_{2}-95 \%$ Ar was then introduced to the catalyst bed. A temperature ramping program with a rate of $10 \mathrm{~K} \mathrm{~min}^{-1}$ was performed after the baseline of mass spectra was stable. A $5 \mathrm{~A}$ zeolite trap was used to remove moisture and water. The hydrogen consumption rate was monitored by mass spectrometry.

\subsection{Catalytic performance}

The DMO hydrogenation reaction was tested in a stainless steel tubular reactor equipped with a computer-controlled auto-sampling system in a continuous flow mode. Typically, $100 \mathrm{mg}$ of the catalyst precursor (40-60 meshes) was sandwiched with quartz powder (40-60 meshes) and loaded in the center of the reactor. Before the catalytic performance was started, all Ag-based precursors were pre-reduced under a 5\%
$\mathrm{H}_{2}-95 \% \mathrm{~N}_{2}$ atmosphere at $623 \mathrm{~K}$ for $4 \mathrm{~h}$ with ramping rates of $2 \mathrm{~K} \mathrm{~min}^{-1}$. The catalyst bed was naturally cooled to $493 \mathrm{~K}$, and pure $\mathrm{H}_{2}$ was fed into the reactor. The system pressure was maintained at 3.0 MPa with the aid of a back-pressure regulator. Subsequently, $0.02 \mathrm{~g} \mathrm{~mL}^{-1}$ DMO methanol solution was pumped into the catalyst bed with a Series III digital HPLC pump (Scientific Systems, Inc.). The products were analyzed on-line using GC7890-MS5875C. The DMO conversion for the calculation of the reaction rates at different reaction temperatures was around $50 \%$. The apparent activation energy was obtained by the Arrhenius equation.

\section{Results and discussion}

\subsection{Comparison of $\mathrm{Ag}$-based materials}

Support and size effects are important issues when comparing different materials for heterogeneous catalysis, especially for structure-sensitive reactions. The support carries the nanocrystallite and directly affects the morphology and particle size of the active site. Moreover, the support may also influence substrate sorption because of different properties, such as surface acid-base properties. When the interaction between metal nanocrystallite and the support is weak, the morphology of the nanocrystallite is always spherical with a large size. ${ }^{20}$ Otherwise, nanocrystallites may be angular and present a small crystallite size. The crystallite size directly accelerates the number of active sites and, consequently, the catalytic ability. ${ }^{21}$ In general, catalysts with smaller crystallite sizes possess better catalytic activity and reaction rate.

Hydrogenation of DMO involves successive hydrogenolysis and hydrogenation of $\mathrm{C}-\mathrm{O}$ bonds, mainly producing several important chemicals, such as MG, EG, and ethanol (EtOH) at different levels. The reaction scope is shown in Table 1. Previous studies found that the performance of DMO hydrogenation is dependent on the $\mathrm{Ag}$ size when the crystallite size is larger than $3.6 \mathrm{~nm}$. Using non-carbon materials as supports,

Table 1 Scheme of DMO hydrogenation, different carrier-supported Ag catalysts, and their performance

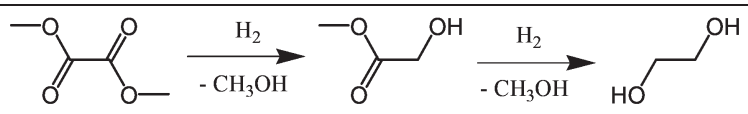

\begin{tabular}{|c|c|c|c|c|c|}
\hline \multirow[b]{2}{*}{ Carrier } & \multirow[b]{2}{*}{$\operatorname{Size}^{a}(\mathrm{~nm})$} & \multirow[b]{2}{*}{$S_{\mathrm{BET}}\left(\mathrm{m}^{2} \mathrm{~g}^{-1}\right)$} & \multirow[b]{2}{*}{$R^{b}\left(\mathrm{mmol} \mathrm{h}^{-1}\right)$} & \multicolumn{2}{|c|}{ Yield (\%) } \\
\hline & & & & MG & EG \\
\hline$\gamma-\mathrm{Al}_{2} \mathrm{O}_{3}$ & 5 & 186 & 46.5 & 37.7 & 39.1 \\
\hline $\mathrm{SiO}_{2}$ & 16 & 312 & 40.7 & 76.5 & 3.7 \\
\hline $\mathrm{H}_{2} \mathrm{SiO}_{3}$ & 4 & 201 & 46.9 & 56.9 & 35.4 \\
\hline $\mathrm{AC}^{c}$ & 7 & 96 & 7.5 & 14.6 & 0 \\
\hline $\mathrm{GA}^{d}$ & $\mathrm{nd}^{f}$ & 18 & 5.0 & 9.8 & 0 \\
\hline $\mathrm{CNF}^{e}$ & nd & 5 & 1.8 & 3.5 & 0 \\
\hline CNT & 18 & 145 & 50.3 & 98.9 & 0.1 \\
\hline
\end{tabular}

${ }^{a}$ Crystallite size was calculated by using the Scherrer equation. ${ }^{b} R$ represents the rate of DMO disappearance. ${ }^{c}$ Activated carbon. ${ }^{d}$ Graphene. ${ }^{e}$ Carbon nanofibers. ${ }^{f}$ Not detected. 
$\mathrm{Ag}$ crystallites follow the size effect rule during DMO hydrogenation, which is in accordance with previous studies. ${ }^{17} \mathrm{Ag} /$ $\mathrm{H}_{2} \mathrm{SiO}_{3}$ with the smallest crystallite size of $4 \mathrm{~nm}$ (Fig. S1 $\dagger$ ), exhibited the best reaction rate and product yield of $91.3 \%$ (56.9\% MG and 35.4\% EG). However, we unexpectedly found no discernable correlation between the $\mathrm{Ag}$ crystallite size and the catalytic activity for Ag-based carbon materials. Ag-in/ $h \mathrm{CNT}$ with the largest crystallite size of $18 \mathrm{~nm}$ presented the best activity and selectivity, which were better than those of the $\mathrm{Ag} / \mathrm{H}_{2} \mathrm{SiO}_{3}$ catalyst.

The abnormal phenomenon implied that, in a reduction and oxidation atmosphere of hydrogen and ester, $\mathrm{Ag}$ species is not the only decisive factor that affects catalytic performance. The choice of solid carrier used may significantly influence the catalytic activity. Therefore, the support effect should be considered as an emergent issue for this reaction. A providential support possessing a large surface area, suitable pore structure, and appropriate surface acidity is an ideal carrier for DMO hydrogenation. The use of acidic $\mathrm{H}_{2} \mathrm{SiO}_{3}$ and basic $\mathrm{Al}_{2} \mathrm{O}_{3}$ as supports for $\mathrm{Ag}$ catalysts cannot achieve good selectivity. However, high MG selectivity was preserved for Ag-supported inert carbon materials and silica oxides. The results indirectly demonstrated that carriers may affect the absorption of the substrate and cause differences in product selectivity. When considering the absorption properties of support materials, the size effect will be indistinct. Among various carbon material-supported Ag, CNT-supported Ag with the largest crystallite size showed superior catalytic activity. The specificity of CNTs should be carefully considered. CNTs possess a large external surface with oxidized vacancies and localized double bonds, electron transfer between the metal and CNTs, special ability in hydrogen storage, confined space of the nanochannel, relatively low limitations for mass transfer, and high thermal conductivity. ${ }^{22}$ Given their intrinsic features, CNTs play a beneficial role in numerous catalytic applications. ${ }^{6-13}$

\subsection{Morphology of Ag-in/hCNT and Ag-out/hCNT and their catalytic performance}

The morphologies of Ag located in or out of the CNT hybrid channels were investigated by TEM. Interestingly, in the case of the Ag-in/hCNT catalyst, an estimated $80 \%$ of the observed $\mathrm{Ag}$ NPs was found in the internal channels of CNTs in long wire shapes, as observed from the TEM snapshots (Fig. 1a). Large Ag crystallites were restricted and had to block the channels, further reducing the exposed surface. In theory, this blocking of $\mathrm{Ag}$ crystallites should present harsh internal diffusion limitations and minimal active surface area. Therefore, regardless of the view of mass transfer or point of size effect, the pore-plugging Ag-in/hCNT catalyst should exhibit low catalytic activity, which was contrary to the experimental results.

By contrast, Ag crystallites implanted on the external surface of CNTs (Fig. 1b) were prepared by the classical excessive impregnation method. Ag crystallites grew on the external surface without confinement into CNTs. The average particle size was calculated from XRD based on the Scherrer equation.
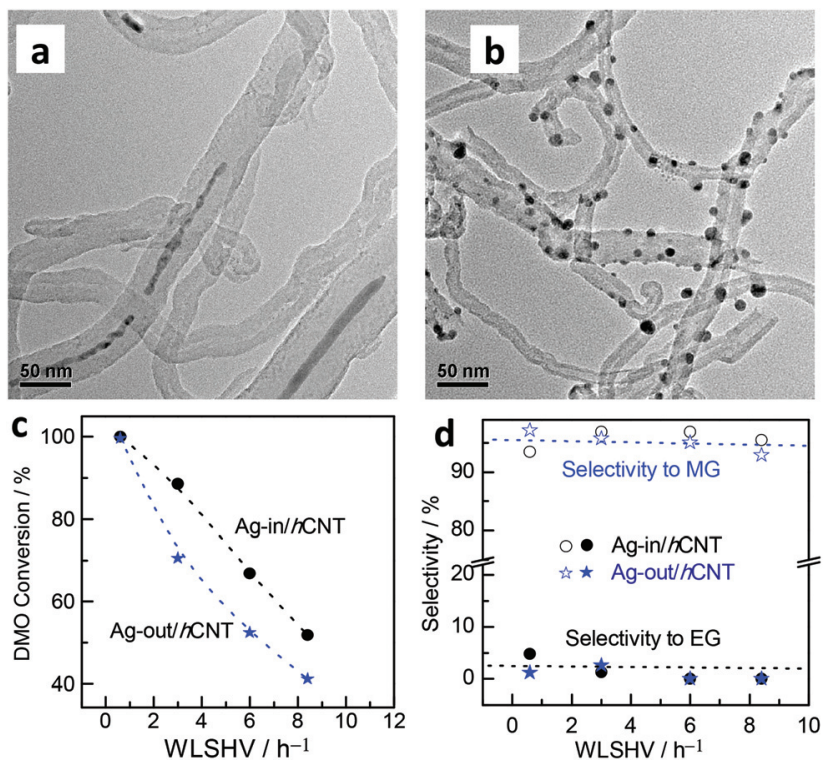

Fig. 1 TEM images of (a) Ag-in/hCNT and (b) Ag-out/hCNT; (c) conversion of DMO and (d) MG selectivity using Ag catalysts.

Ag-out/hCNT catalysts possess a smaller crystallite Ag size of $11 \mathrm{~nm}$, whereas the Ag crystallite in the Ag-in/hCNT catalyst is approximately $18 \mathrm{~nm}$. This value cannot conclude the accurate specification of Ag nanowires, but we can calculate the specification by translating the nanowires into a spherical model. Based on $4 / 3 \times \pi r^{3}=L \times \pi(d / 2)^{2}$, where the spherical radius $(r)$ is $9 \mathrm{~nm}$ and the CNT channel size $(d)$ is about $8 \mathrm{~nm}$, the average nanowire length $(L)$ is obtained as $60 \mathrm{~nm}$. In addition, the morphology of most Ag crystallites is spherical, indicating that the interaction between outer Ag and CNT is weak. By contrast, confined Ag, which is shaped into wires, may strengthen the interaction of $\mathrm{Ag}$ and CNTs.

However, abnormal activity was observed (Fig. 1c). At a weight liquid hourly space velocity (WLHSV) of $0.6 \mathrm{~h}^{-1}$, both catalysts exhibited an ideal performance, with $100 \%$ conversion of DMO. Ag crystallites confined inside the channel of CNTs displayed a higher level of conversion and reaction rate compared with Ag-out/hCNT under elevated LHSV. However, activity tests showed that MG was the dominant product (Fig. 1d), regardless of whether Ag crystallites were in or out of CNTs. Therefore, minimal internal diffusion limitations existed to inhibit the process of chemoselective hydrogenation of DMO to MG. Considering that high conversion was obtained over $\mathrm{Ag}$-in/hCNT, the hydrogenation of $\mathrm{C}=\mathrm{O}$ bonds should mainly occur on the outer surface of CNTs so that the reaction could proceed, regardless of the occurrence of pore plugging.

\subsection{Ability to activate dihydrogen}

Although hydrogen is not prone to forming bonds with $\mathrm{Ag}$ as the d-orbital of $\mathrm{Ag}$ is filled, ${ }^{23}$ especially for larger $\mathrm{Ag}$ nanocrystallites with few defects and grain boundaries, the metallic $\mathrm{Ag}$ could absorb hydrogen in our case, rendering $\mathrm{C}=\mathrm{O}$ hydro- 
genation reaction possible. The electronic structure of metallic $\mathrm{Ag}$ should be subtly modified. $\mathrm{H}_{2}$ chemisorption (Fig. 2a) was conducted to compare the activation ability of $\mathrm{Ag}$ dependent on the located position relative to CNTs. Notably, Ag confined inside CNTs possessed almost twice the amount of $\mathrm{H}_{2}$ sorption than that of outside Ag. We further used Arrhenius plots to derive apparent activation energies ( $\left.E_{\mathrm{a}}\right)$ (Fig. 2b). $E_{\mathrm{a}}$ was $349 \mathrm{~kJ} \mathrm{~mol}^{-1}$ for Ag-out/hCNT, whereas the value for Ag-in/ $h \mathrm{CNT}$ was approximately $221 \mathrm{~kJ} \mathrm{~mol}^{-1}$. Ag located inside the channel of CNTs benefits more for $\mathrm{H}_{2}$ activation, which is crucial for hydrogenation reactions. The confined environment can enrich the reactant atmosphere. ${ }^{6}$ In our case, $\mathrm{H}_{2}$ may be concentrated inside the channels of CNTs, facilitating the hydrogenation reaction toward hydroproducts. Both $\mathrm{H}_{2}$ chemisorption and apparent activation energies revealed that confined $\mathrm{Ag}$ possessed stronger ability to activate dihydrogen and facilitated hydrogenation.

\subsection{Probe into the texture structure for $\mathrm{H}_{2}$ activation}

As shown in Fig. 1 and 3, confined Ag NPs were oppressed and stretched into wires by the interior wall of CNTs, whereas free $\mathrm{Ag}$ atoms integrated into spherical particles on the exterior wall of CNTs. As sketched from high-resolution TEM, both Ag nanowires inside CNTs and Ag particles outside CNTs possessed grain boundaries, in which atom vacancies were enriched. The former exhibited a rougher surface, with more grain boundaries. Balluffi and Seigle showed that sufficient vacancies can be produced at transverse grain boundaries because of the tension acting across the boundary. ${ }^{24}$ The stress from the interior walls of CNTs and the confined environment cause the stretching of $\mathrm{Ag}$ particles, leading to the emergence of atom dislocation and the consequent formation of grain boundaries and vacancies. Grain boundaries are recommended as rapid diffusion paths for hydrogen species, and atomic hydrogen species will emerge and transfer onto vacancies. The activation of $\mathrm{H}_{2}$ is further improved at accessorial vacancies in the grain boundaries. We previously proved that metallic $\mathrm{Ag}$ is structurally sensitive to the activity of DMO hydrogenation. Vacancies can be regarded as open volume

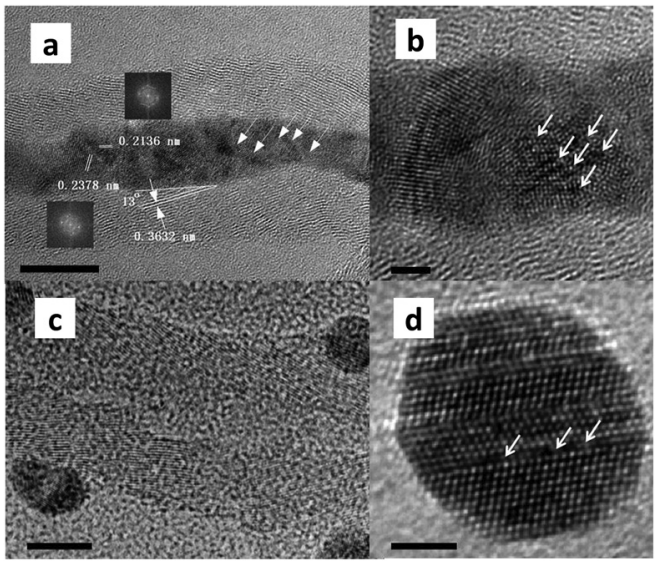

Fig. 3 High-resolution TEM images of (a) and (b) Ag-in/hCNT; (c) and (d) Ag-out/hCNT. The bar of (a) and (c) is 10 and $2 \mathrm{~mm}$ for (b) and (d). The inserted figures show the FFT changes of Ag crystallites and were used to calculate lattice spacing. The arrows show the vacancies in $\mathrm{Ag}$ crystallites.

defects and are able to trap hydrogen species. ${ }^{25}$ The concentrated hydrogen species is beneficial for DMO hydrogenation. This finding can explain why Ag-in/hCNT possesses stronger $\mathrm{H}_{2}$ chemisorption and reduced activation energy barrier.

\subsection{Approach of $\mathbf{H}_{2}$ diffusion}

However, the mechanism underlying the reaction of activated hydrogen species on the Ag nanowires with DMO molecule remains unclear. Given that no entries are available for the diffusion of a comparatively larger molecule DMO, we can easily speculate that CNTs should be able to connect the activated hydrogen species and the atmospheric DMO. Previous reports revealed that carbon materials play an important role in $\mathrm{H}_{2}$ transfer and spillover. ${ }^{26-29}$ Both theoretical and experimental studies have demonstrated that activated hydrogen species derived from dihydrogen can flow onto graphitic carbon surfaces proximal to a metallic subnanoparticle and subsequently diffuse to other sites of the materials. ${ }^{29}$ Yang used carbon materials to bridge the metal and the receptor to
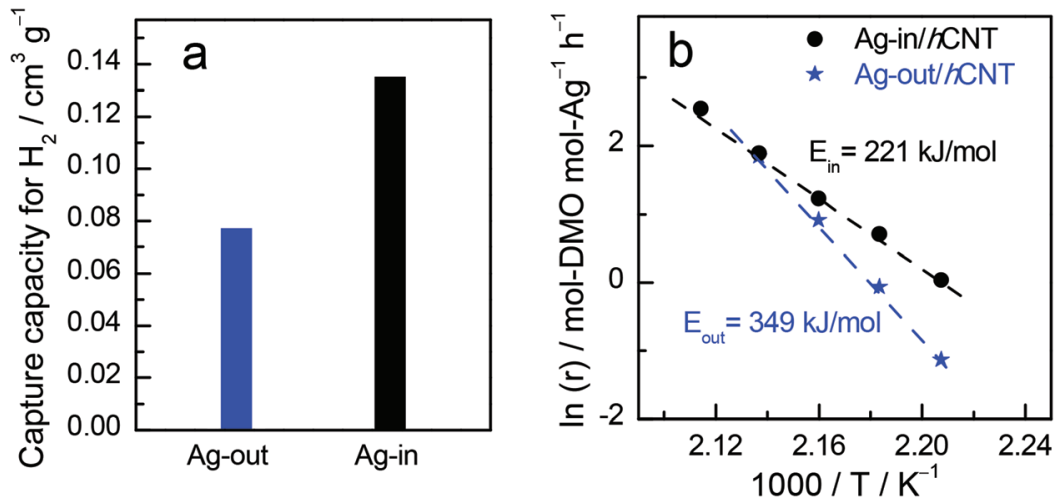

Fig. 2 Comparison of (a) $\mathrm{H}_{2}$ chemisorption and (b) apparent activation energies. The symbol $r$ means the reaction rate, moles of DMO converted by per mole of Ag per hour (mol-DMO per mol-Ag per $\mathrm{h}$ ). 
enhance hydrogen spillover and depicted that activated hydrogen species can be transferred and stored in the interval layer of graphitic sheets. ${ }^{27}$ Among the different kinds of carbon materials, multi-walled CNTs possess rolled-up graphitic layers and are classified as parallel-type and herringbone-type on the basis of the rotational axis. As exhibited by high-resolution TEM, the graphitic layers of Ag-out/hCNT and Ag-in/hCNT were tilted by $13^{\circ}$ relative to the one-dimensional tunnel axis, demonstrating the herringbone structure of CNTs with $0.36 \mathrm{~nm}$ of interlamellar spacing. The terminals of these graphitic layers were in intimate contact with $\mathrm{Ag}$ nanowires for $\mathrm{Ag}$-in/hCNT. Thus, the activated hydrogen atoms could migrate from confined $\mathrm{Ag}$ nanowires into the interlayer, thereby rendering the activated hydrogen atoms available to react with DMO molecules. To confirm that the internal activated hydrogen atoms on $\mathrm{Ag}$ nanowires could approach the external receptor DMO through the interlayer in the herringbone CNTs, we deliberately adopted two designed experiments.

As the first strategy, Ag nanowires confined in the pores of parallel CNTs and SBA-15 (Fig. 4) were evaluated for comparison. Notably, confined $\mathrm{Ag}$ in parallel CNTs (Ag-in/ $p \mathrm{CNT})$ showed an $R$ value of $14.1 \mathrm{mmol} \mathrm{h}^{-1}$ for the DMO hydrogenation. This activity was considerably lower than the activity of $\mathrm{Ag}$ located in the herringbone CNTs. The major difference of these two catalysts was the architectural structure of CNTs. Although atomic hydrogen species can diffuse and be stored in interplanar graphitic layers, the species can hardly pass through graphitic layers with only $0.142 \mathrm{~nm}$ of carbon-carbon bonds, unless on the domain enriched with defect sites. Chen et al. investigated the hydrogen-migrated behavior using $\mathrm{KOH}$ to partially etch graphitic carbon on the surfaces of parallel CNTs. ${ }^{30}$ They found that hydrogen atoms easily diffuse and migrate from the external surface into the interplanar spacing

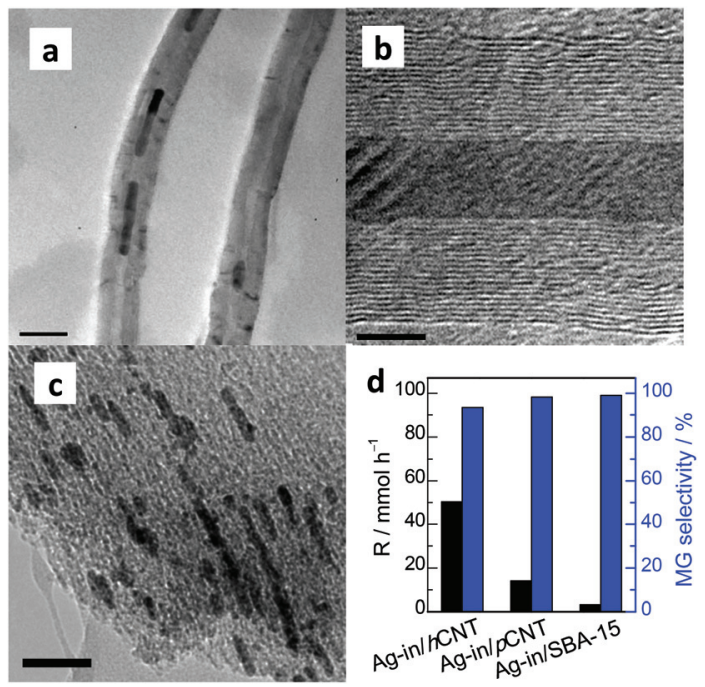

Fig. 4 (a) TEM images of parallel CNT confined Ag nanowires. (b) Highresolution TEM of $\mathrm{Ag}$-in/pCNT and (c) Ag-in/SBA-15. (d) Performance of herringbone CNTs, parallel CNTs, and SBA-15 confined Ag catalysts. The bar of (a) and (c) is $50 \mathrm{~nm}$ and (b) is $5 \mathrm{~nm}$. via defect sites on the surface of activated CNTs, causing hydrogen spillover enhancement. Therefore, the diffusion of activated hydrogen atoms on $\mathrm{Ag}$ nanowires in parallel CNTs was tightly strained by the multiple walls of graphitic layers, resulting in poor hydrogenation performance. On the contrary, activated hydrogen atoms on $\mathrm{Ag}$ nanowires could directly migrate through the terminal of the epitaxial interplanar space. The enhanced performance insinuated that the herringbone CNTs exhibited a better capacity for hydrogen spillover than parallel CNTs. In addition, we speculated that the stored hydrogen species in the interplanar space of CNTs could be further utilized for the hydrogenation of DMO. Although the comparison of herringbone CNTs and parallel CNTs is barely discussed, herringbone CNTs are recognized as a better support in most cases that have appeared. ${ }^{31}$ SBA-15 is a wellknown inert support with low acidity that possesses $2 \mathrm{D}$ channels, but the silica layers are impenetrable. As shown in Fig. 4c, Ag nanowires blocked the channels of SBA-15, which may extremely limit the activation of dihydrogen and the diffusion of activated hydrogen species and DMO. Thus, Ag nanowires in SBA-15 predictably showed only trace activity. This finding also indicated that carbon medium material was necessary for hydrogen diffusion and spillover for the confined Ag nanowires.

Activated hydrogen species can be transferred and stored in the interplanar space. However, whether stored hydrogen will tarry in the interplanar space or diffuse to the outer surface and be used for hydrogenation remains unsure. For confirmation, we prepared Cu-out-Ag-in/hCNT (Fig. 5b) loaded with copper NPs onto the external surface of Ag-in/hCNT catalysts. The TPR technique (Fig. 5c) was employed to investigate the reductive behavior of outside copper NPs, and a mass spectrometer was used as a detector to ensure accuracy. The result showed that Ag-in/hCNT presented negligible reductive signal, indicating the spontaneous reductive behavior of $\mathrm{Ag}$ crystallites supported on CNTs during calcination. Similar spontaneous reductive phenomena have been frequently observed for numerous noble metals supported on CNTs. ${ }^{32,33}$ Meanwhile, copper NPs supported on CNTs maintained the oxidative state and showed a reductive peak maximum at $538 \mathrm{~K}$. Interestingly, the reductive peak shifted to $514 \mathrm{~K}$ when $\mathrm{Ag}$ nanowires were introduced into CNTs. Copper NPs supported on Ag-in/hCNT could be easily reduced compared with copper NPs carried by pure CNTs. Hydrogen species detained in the interplanar space of CNTs could diffuse to the outer copper NPs through the terminal and reduce them. ${ }^{6}$ Moreover, hydrogen species activated by Ag-in/hCNT could be further utilized for the reduction of copper NPs located on the external surface of CNTs, although the Ag nanowires and copper NPs were separated by the multi-walled CNTs.

\subsection{Plausible scheme of confinement and promotional effects via CNT intermediate confined $\mathrm{Ag}$}

The possible confinement and promotional effects are compared in Scheme 1. On the basis of the above analysis, the confinement effect caused compressive deformation for $\mathrm{Ag}$ 

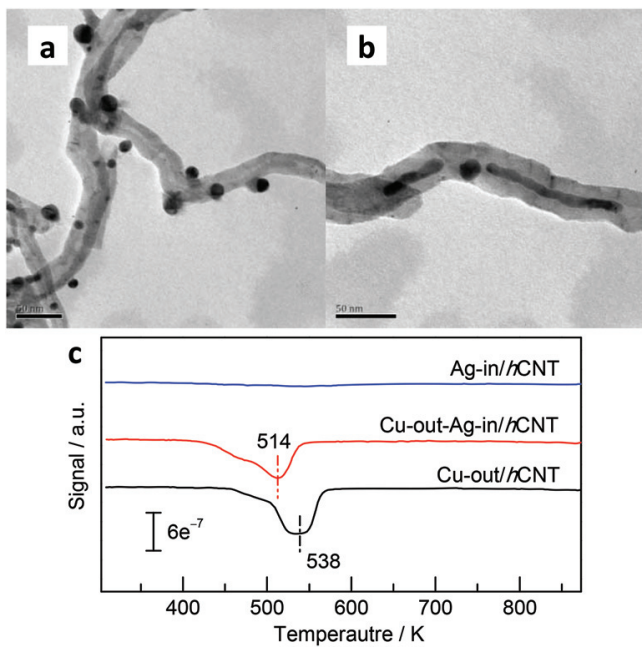

Fig. 5 TEM images of (a) Cu-out/hCNT and (b) Cu-out-Ag-in/hCNT. (c) TPR profiles of Ag-in/hCNT, Cu-out/hCNT, and Cu-out-Ag-in/hCNT.

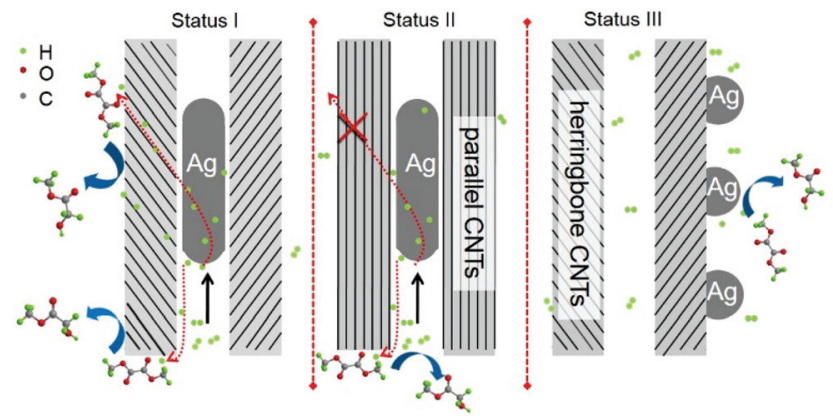

Scheme 1 Plausible scheme for the confinement and promotional effects of $\mathrm{Ag} / \mathrm{C}$ hybrids.

crystallite confined in the internal channel of CNTs. As a result, Ag crystallites were shaped in wires containing grain boundaries with abundant vacancies. Despite the weak interaction of $\mathrm{Ag}$ and dihydrogen, activation of dihydrogen could be further enhanced on $\mathrm{Ag}$ vacancies, and grain boundaries could provide approaches for hydrogen diffusion. Interactions may be developed between encapsulated nanomaterials and CNT surfaces. The curvature of CNT walls is reported to cause the $\pi$ electron density of graphene layers to shift from the concave inner surfaces to the convex outer surface, resulting in an electric potential difference. ${ }^{34,35}$ As a result, the molecules and nanomaterials on the exterior walls of CNTs probably displayed different properties and chemical reactivity from those confined within CNTs. According to the different electronegativities of $\mathrm{Ag}$ and carbon (C) (1.93 versus 2.55), interactions of $\mathrm{Ag}$ and $\mathrm{C}$ may cause electron transfer from metallic $\mathrm{Ag}$ to CNTs, resulting in electron-deficient Ag crystallites. The curvature of CNTs may cause the interior walls to become more electrophilic, thereby aggravating the electron-deficient properties, even for the herringbone CNTs, which had interior walls that leaned to the internal $\mathrm{Ag}$ nanowires. Experiments and DFT calculations have proven that electron-deficient metallic sites are beneficial for the activation of dihydrogen. ${ }^{36,37}$ With the combination of geometric and electronic effects, the confined Ag should present stronger capacity to activate dihydrogen. This finding has been confirmed by $\mathrm{H}_{2}$ chemisorption characterization and apparent activation energy tests.

It was reported that the electrons of the encapsulated $\mathrm{CoNi}$ clusters can penetrate through three covered parallel graphene layers, making hydrogen species adsorption available on covered graphene shells. ${ }^{38}$ Calculations showed that the electronic potential difference decreased from $-0.5 \mathrm{eV}$ to $0 \mathrm{eV}$ when the graphene cover increased from one to three layers. Thus, the effect of enclosed metals in graphene shells consisting of more than three layers will decline. In the present work, the electronic penetration effect of Ag-in $/ p$ CNT with approximately 20 layers of graphite was probably suppressed and negligible. The activation of dihydrogen may be influenced by the shape of nanomaterials and the activated hydrogen species may facilitate the hydrogenation reaction through hydrogen spillover. In contrast to parallel CNTs, the herringbone CNTs obtained showed not only the confinement effect but also the relatively exoteric access of activated hydrogen species. Design experiments proved that activated hydrogen species on $\mathrm{Ag}$ nanowires in the channels of herringbone CNTs could be transferred and utilized by the outer surface species. By contrast, activated hydrogen species in the channel of parallel CNTs could hardly transfer to the outer space, except back to the original inlet. Predictably, the direct contact of interplanar spacing terminals to $\mathrm{Ag}$ species offered an approach for hydrogen diffusion. Based on the previous literature, ${ }^{34,35}$ the CNT surface is beneficial for the $\pi-\pi$ interaction between $\mathrm{C}=\mathrm{O}$ bonds of DMO and the basic $\pi$ sites on CNT surfaces, enabling the activated hydrogen species to diffuse from the internal Ag to hydrogenate the carbonyl group of DMO. Life test (Fig. 6) was conducted and it was observed that the activity of Ag-in $/ h$ CNT maintained at a high MG yield $>97 \%$ for more than $200 \mathrm{~h}$, revealing high catalytic stability. This indicated that the performance aided by the con-

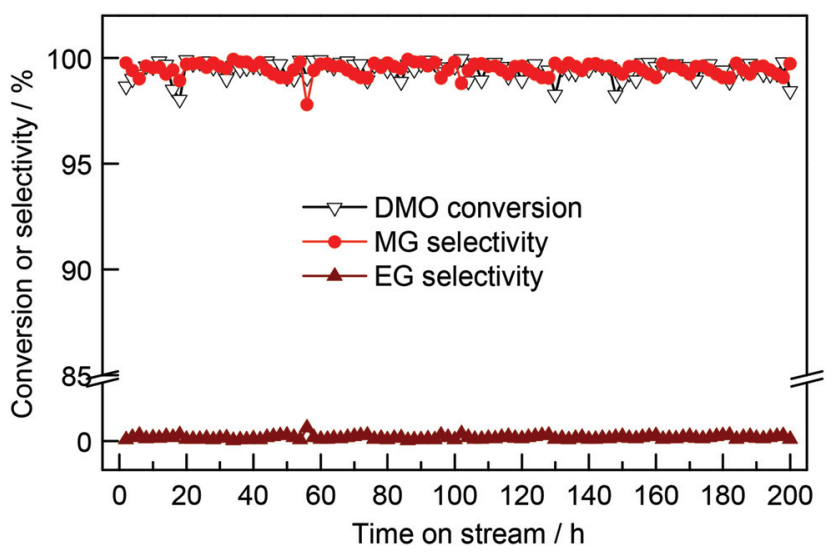

Fig. 6 Catalytic performance of $\mathrm{Ag}$-in/hCNT as a function of reaction time. Reaction conditions: $T=493 \mathrm{~K}, P\left(\mathrm{H}_{2}\right)=3.0 \mathrm{MPa}, \mathrm{H}_{2} / \mathrm{DMO}=80$, WLHSV $=0.6 \mathrm{~h}^{-1}$. 
finement effect and promotional effect could last and the Ag-in/ $h$ CNT catalyst showed a potential industrial application for MG synthesis from DMO hydrogenation.

\section{Conclusions}

In conclusion, herringbone CNT confined Ag NPs with nanowire-like morphology, Ag-in/hCNTs, were first prepared and found to exhibit superior catalytic performance for DMO hydrogenation to MG. The Ag-in/hCNT catalyst exhibited approximately three times higher activity than Ag NPs located on the CNT exterior walls (Ag-out/hCNT) for DMO hydrogenation. Moreover, Ag confined in the channel showed excellent durability over $200 \mathrm{~h}$ with high MG yield greater than $97 \%$. High-resolution TEM showed that confined Ag crystallites were shaped into wires, whereas free Ag particles were spherical, indicating the stronger interaction between confined Ag nanocrystals and CNTs. The confinement effect was found to induce a geometric effect, which made the surface of $\mathrm{Ag}$ rough and enriched with grain boundaries and vacancies. These grain boundaries and vacancies may be beneficial for the activation and diffusion of hydrogen species, helping in increasing the chemisorption of hydrogen and decreasing the apparent activated energy. The activated hydrogen could diffuse to the interplanar spaces of herringbone CNTs, as verified by the promoted reduction of copper NPs on the external surface of herringbone CNTs. Subsequently, hydrogen diffusion from confined $\mathrm{Ag}$ to herringbone CNTs could implement the hydrogenation of DMO. This work provides protocols to realize confined $\mathrm{Ag}$ within CNTs and understand the active hydrogen spillover (migration), benefiting fundamental research on hydrogenation and industrial applications for MG synthesis.

\section{Acknowledgements}

We acknowledge the financial support from the Natural Science Foundation of China (91545115, 21473145, 21403178, and 21303141), the Postgraduate Basic Innovative Research Program of Xiamen University (201412G001), and the Program for Innovative Research Team in Chinese Universities (no. IRT_14R31).

\section{Notes and references}

1 A. Y. Yin, X. Y. Guo, W. L. Dai and K. N. Fan, Chem. Commun., 2010, 46, 4348.

2 H. H. Liu, G. K. Chuah and S. Jaenicke, Phys. Chem. Chem. Phys., 2015, 17, 15012.

3 C. Gao, W. W. Li, Y. Z. Jin and H. Kong, Nanotechnology, 2006, 17, 2882.

4 P. Luksirikul, K. Tedsree, M. G. Moloney, M. L. Green and S. C. Tsang, Angew. Chem., Int. Ed., 2012, 51, 6998.

5 P. Zhang, C. L. Shao, Z. Y. Zhang, M. Y. Zhang, J. B. Mu, Z. C. Guo and Y. C. Liu, Nanoscale, 2011, 3, 3357.
6 X. L. Pan and X. H. Bao, Acc. Chem. Res., 2011, 44, 553.

7 S. J. Guo, X. L. Pan, H. L. Gao, Z. Q. Yang, J. J. Zhao and X. H. Bao, Chem. - Eur. J., 2010, 16, 5379.

8 X. L. Pan, Z. L. Fan, W. Chen, Y. J. Ding, H. Y. Luo and X. H. Bao, Nat. Mater., 2007, 6, 507.

9 E. Castillejos, R. Chico, R. Bacsa, S. Coco, P. Espinet, M. Pérez-Cadenas, A. Guerrero-Ruiz, I. Rodríguez-Ramos and P. Serp, Eur. J. Inorg. Chem., 2010, 5096.

10 W. Chen, Z. L. Fan, X. L. Pan and X. H. Bao, J. Am. Chem. Soc., 2008, 130, 9414.

11 A. M. Zhang, J. L. Dong, Q. H. Xu, H. K. Rhee and X. L. Li, Catal. Today, 2004, 93-95, 347.

12 J.-P. Tessonnier, L. Pesant, G. Ehret, M. J. Ledoux and C. Pham-Huu, Appl. Catal., A, 2005, 288, 203.

13 L. Zhang, L. P. Han, G. F. Zhao, R. J. Chai, Q. F. Zhang, Y. Liu and Y. Lu, Chem. Commun., 2015, 51, 10547.

14 J. W. Zheng, J. F. Zhou, H. Q. Lin, X. P. Duan, C. T. Williams and Y. Z. Yuan, J. Phys. Chem. C, 2015, 119, 13758.

15 C. Wen, Y. Y. Cui, W. L. Dai, S. Xie and K. N. Fan, Chem. Commun., 2013, 49, 5195.

16 H. R. Yue, Y. J. Zhao, S. Zhao, B. Wang, X. B. Ma and J. L. Gong, Nat. Commun., 2013, 4, 2339.

17 J. W. Zheng, H. Q. Lin, X. L. Zheng, X. P. Duan and Y. Z. Yuan, Catal. Commun., 2013, 40, 129.

18 A. Y. Yin, C. Wen, W. L. Dai and K. N. Fan, Appl. Catal., B, 2011, 108-109, 90.

19 B. Xue, P. Chen, Q. Hong, J. Y. Lin and K. L. Tan, J. Mater. Chem., 2001, 11, 2378.

20 J. Y. Liu, ChemCatChem, 2011, 3, 934.

21 J. Guerra and M. A. Herrero, Nanoscale, 2010, 2, 1390.

22 R. Kumar, E. Gravel, A. Hagege, H. Li, D. V. Jawale, D. Verma, I. N. Namboothiri and E. Doris, Nanoscale, 2013, 5, 6491.

23 G. Vilé, D. Baudouin, I. N. Remediakis, C. Copéret, N. López and J. Pérez-Ramírez, ChemCatChem, 2013, 5, 3750 .

24 R. W. Balluffi and L. L. Seigle, Acta Metall., 1957, 5, 449.

25 A. Pundt and R. Kirchheim, Annu. Rev. Mater. Res., 2006, $36,555$.

26 Y. W. Li and R. T. Yang, J. Am. Chem. Soc., 2006, 128, 8136.

27 A. J. Lachawiec, J. G. S. Qi and R. T. Yang, Langmuir, 2005, 21, 11418.

28 L. F. Wang and R. T. Yang, Catal. Rev., 2010, 52, 411.

29 L. Chen, A. C. Cooper, G. P. Pez and H. S. Cheng, J. Phys. Chem. C, 2007, 111, 18995.

30 C. H. Chen and C. C. Huang, Microporous Mesoporous Mater., 2008, 109, 549.

31 X. L. Liang, X. Dong, G. D. Lin and H. B. Zhang, Appl. Catal., B, 2009, 88, 315.

32 B. M. Quinn, C. Dekker and S. G. Lemay, J. Am. Chem. Soc., 2005, 127, 6146.

33 P. Singh, G. Lamanna, C. Menard-Moyon, F. M. Toma, E. Magnano, F. Bondino, M. Prato, S. Verma and A. Bianco, Angew. Chem., Int. Ed., 2011, 50, 9893. 
34 Z. Guo, Y. T. Chen, L. S. Li, X. M. Wang, G. L. Haller and Y. Yang, J. Catal., 2010, 276, 314.

35 Y. B. Yan, J. W. Miao, Z. H. Yang, F. X. Xiao, H. B. Yang, B. Liu and Y. Yang, Chem. Soc. Rev., 2015, 44, 3295.

36 M. Bron, D. Teschner, A. Knop-Gericke, F. C. Jentoft, J. Krohnert, J. Hohmeyer, C. Volckmar, B. Steinhauer,
R. Schlogl and P. Claus, Phys. Chem. Chem. Phys., 2007, 9, 3559.

37 Y. Xu, J. Greeley and M. Mavrikakis, J. Am. Chem. Soc., 2005, 127, 12823.

38 J. Deng, P. J. Ren, D. H. Deng and X. H. Bao, Angew. Chem., Int. Ed., 2015, 54, 2100. 